\title{
Effects of Hallux Valgus Orthoses on Ground Reaction Force Using 3D Motion Analysis in Individuals With Hallux Valgus Deformity
}

\author{
Yong-wook Kim, PT, PhD \\ Department of Physical Therapy, College of Medical Sciences, Jeonju University, Jeonju, Korea
}

\author{
Article Info \\ Received September 23, 2020 \\ Revised October 20, 2020 \\ Accepted October 20, 2020 \\ Corresponding Author \\ Yong-wook Kim \\ E-mail: ptkim@jj.ac.kr \\ https://orcid.org/0000-0002-0311-9766
}

\section{Key Words}

Foot orthoses

Hallux valgus

Kinetics

\begin{abstract}
Background: Hallux valgus (HV) is a foot deformity developed by mediolateral deviation of the first metatarsophalangeal joint. Although various foot-toe orthoses were used to correct the HV angle, verification of the effects of kinetics variables such as ground reaction force (GRF) through three-dimensional (3D) gait analysis according to the various type of orthoses for HV is insufficient.
\end{abstract}

Objects: This study aimed to investigate the effect of soft and hard types of foot and toe orthoses to correct HV deformity on the GRF in individuals with HV using 3D motion analysis system during walking.

Methods: Twenty-six subjects participated in the experiment. Participants had HV angle of more than $15^{\circ}$ in both feet. Two force platforms were used to obtain 3D GRF data for both feet and a 3D motion capture system with six infrared cameras was used to measure exact stance phase point such as heel strike or toe off period. Total walk trials of each participant were 8 to 10, the walkway length was $6 \mathrm{~m}$. Two-way repeated measures ANOVA was used to determine the effects of each orthosis condition on the various GRF values.

Results: The late anteroposterior maximal force and a first vertical peak force of the GRF showed that the hard type orthosis condition significantly increased GRF compared to the other orthosis conditions ( $p<0.05$ ).

Conclusion: There were significant effects in GRF values when wearing the hard type foot orthosis. However, the hard type foot orthosis was uncomfortable to wear during walking. Therefore, it is necessary to develop a new foot-toe orthosis that can compensate for these disadvantages.

\section{INTRODUCTION}

Hallux valgus deformity (HVD) is one of the most common foot disorders associated with chronic pain, and as the hallux deviates outward, the first metatarsal bone head protrudes inward [1]. When HVD occurs, patients usually complain of bunion pain on the medial head area of the first metatarsal bone, lateral subluxation of the first metatarsophalangeal joint, lateral displacement of the sesamoid bone, and pronation of the hallux [2-4]. The causes of HVD have not yet been clearly identified but can be largely divided into extrinsic and intrinsic causes. The extrinsic causes include wearing high-heeled shoes and long-term weight-bearing on the medial forefoot during standing and walking. The intrinsic causes include genetic causes, sex, ligament relaxation, age, metatarsal shape, flat feet, hallux limits, and shortening of the Achilles tendon [5-7].

Imbalances in the muscle activity of the abductor hallucis and the adductor hallucis during walking are characteristics of patients with HVD [8]. In addition, it has been reported that the center of pressure (COP) in the first metatarsophalangeal joint is moved in the medial direction during the terminal stance in the gait cycle. This medial movement of the COP has been reported to increase hip internal rotation, decrease the toe-out angle, and increase knee deformation [9]. Compared to normal subjects, the gait patterns of patients with HVD showed decreased walking speed and stride length [10], and decreased range of motion of the lower limb joints [11].

The clinical interventions for HVD include therapeutic exercises, foot orthoses, drug therapy, and surgical therapy [1,2,4]. 
Among those, the application of foot and toe orthoses to correct the alignment of the first metatarsophalangeal joint is preferred for minor to moderately severe HVD $[12,13]$. However, previous studies on the effect of foot and toe orthoses in HVD have only verified relatively simple variables such as satisfaction with wearing a foot and toe orthosis or changes in the angle of the big toe spread [11-13]. Thus, verification of the effects of kinetic variables such as the ground reaction force (GRF) through three-dimensional (3D) gait analysis according to the various type of orthoses for HVD is lacking.

Therefore, the purpose of this study was to investigate the effect of soft and hard types of foot and toe orthoses to correct HVD on the GRF in individuals with HVD using a 3D motion analysis system during gait.

\section{MATERIALS AND METHODS}

\section{Subjects}

The participants in this study were 26 adults (five males and 21 females) with HVD. The inclusion criteria were a hallux valgus angle of more than $15^{\circ}$ measured using an universal goniometer in both feet and no severe pain of the hallux area or history of foot surgery. The average hallux valgus angle was $25.3^{\circ} \pm 6.9^{\circ}$ in the right toe and $26.1^{\circ} \pm 7.4^{\circ}$ in the left toe. All participants fully understood the purpose and method of the study, provided written informed consent, and voluntarily participated in this study. The study was approved by the Institutional Review Board of Jeonju University (jjIRB-

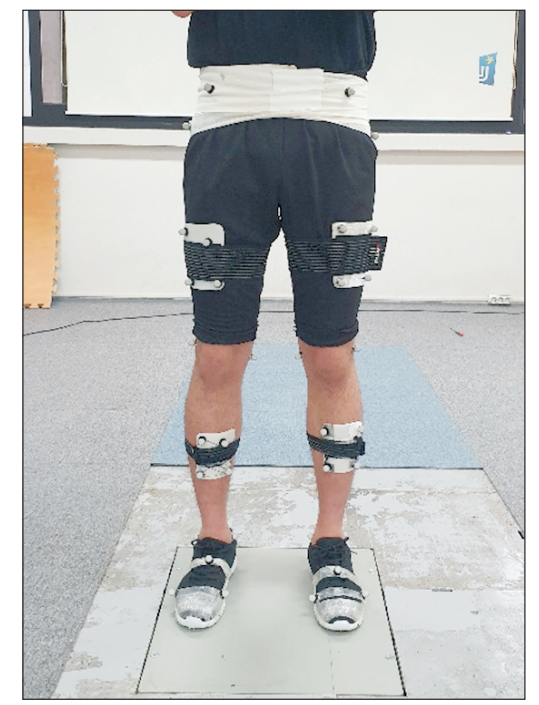

Figure 1. Reflective marker settings on the lower extremities and pelvis during the static calibration and walking trials.
180905-HR-2018-0904). The mean age, height, and weight of all participants were $21.5 \pm 0.8$ years, $164.9 \pm 7.3 \mathrm{~cm}$, and $60.5 \pm 10.1 \mathrm{~kg}$, respectively.

\section{Measurement Devices and Procedure}

Two force platforms (BP400600; AMTI, Watertown, MA, USA) were used to obtain the 3D GRF data while walking in three different foot orthosis conditions (barefoot, soft-type, and hard-type orthoses). A Vicon Motion Capture System (Vicon Inc., Oxford, England) with six infrared cameras (model T10) was used to select the exact gait cycle points such as the heel strike or toe-off moment, and the sampling rate was set at 100 Hz. Nexus 1.8.5 software (Vicon Inc.) was used to process the captured motion and GRF data in the 3D space. A $7.5 \mathrm{~cm}$ Tframe wand was used to calibrate the motion capture system, and a calibration reference object was used to identify the lab origin.

Four cluster reflective markers were attached bilaterally to the thigh and calf segment according to the six-degrees-offreedom (6DOF) model [14]. A total of 40 reflective markers $(1.4 \mathrm{~cm})$ were attached bilaterally to the participant's anterior and posterior superior iliac spine, greater trochanter, femur epicondyle, malleolus, rear feet, mid-feet, and fore-feet (Figure 1). Before the collection of the dynamic walking motion capture data of the lower extremities, static calibration data were obtained from each subject to make a template model for later analysis of the GRF during the walking trials. Following the data collection of static calibration capture, the calibrated anatomical system technique was used to obtain the changes in the kinetic GRF data of the lower extremities while the participants walked freely under the three different foot orthosis
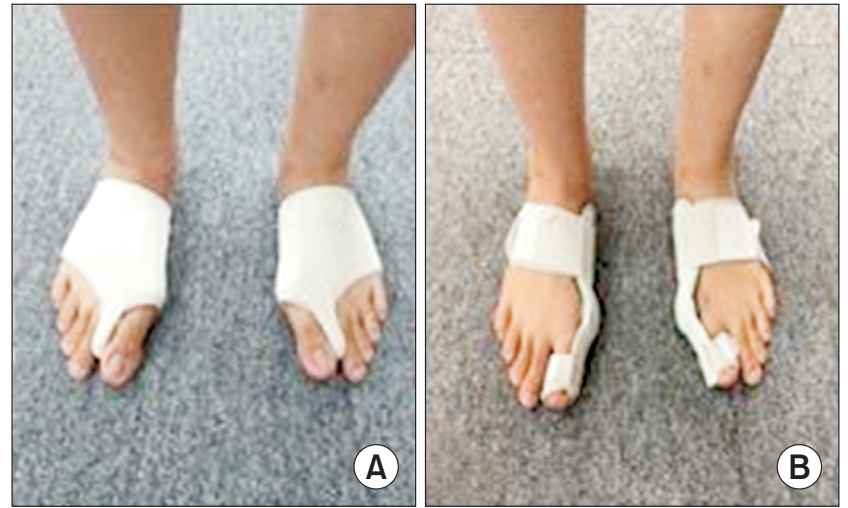

Figure 2. Hallux valgus orthoses. (A) Soft-type orthosis. (B) Hard-type orthosis. 
conditions of a soft-type orthosis (STO), a hard-type orthosis (HTO), and barefoot (BF) (Figure 2). To obtain the dynamic GRF data from two force platforms installed in the middle of the walkway, the participants were asked to walk along a $6 \mathrm{~m}$ walkway in the laboratory at their self-selected speed. A total of 8 to 10 walk trials was collected for each orthosis condition and the average GRF data were obtained bilaterally through all walk trials. The application order of the orthosis conditions was assigned randomly before the experimental trials began.

Following the data collection using the 3D motion capture system and the Vicon Nexus software program, Visual3D analysis software (C-Motion, Rockville, MD, USA) was used to obtain the final GRF results and graph the reports of each joint of the lower limbs. The GRF kinetic data were low-pass filtered with a fourth-order Butterworth filter and a cutoff frequency of $15 \mathrm{~Hz}$. Following the right-hand rule for the segment coordinate system axes, the $\mathrm{X}-\mathrm{Y}-\mathrm{Z}$ Cardan sequence was used to define the order of the rotations [14]. Visual3D produced a visual representation of the magnitudes and directions of the GRF bilaterally in space, which enabled the related stance phase to be calculated (Figures 3 and 4). The GRF data were normalized for body weight.

\section{Data Analysis}

The Kolmogorov-Smirnov test was used to confirm that the GRF data were distributed normally. Two-way repeatedmeasures ANOVA was used to determine the effects of each orthosis condition and foot side. When a significant F-value

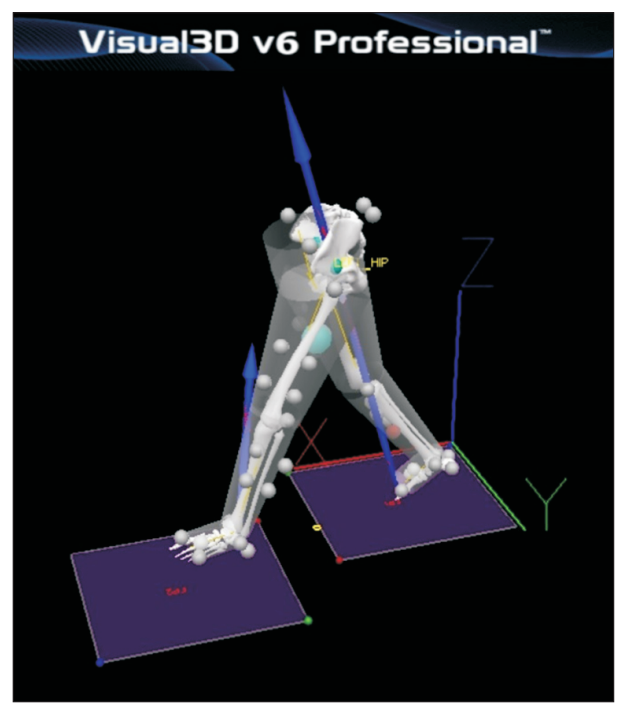

Figure 3. Visual3D representation of the magnitudes and directions of the ground reaction force bilaterally in space during the walking trial.
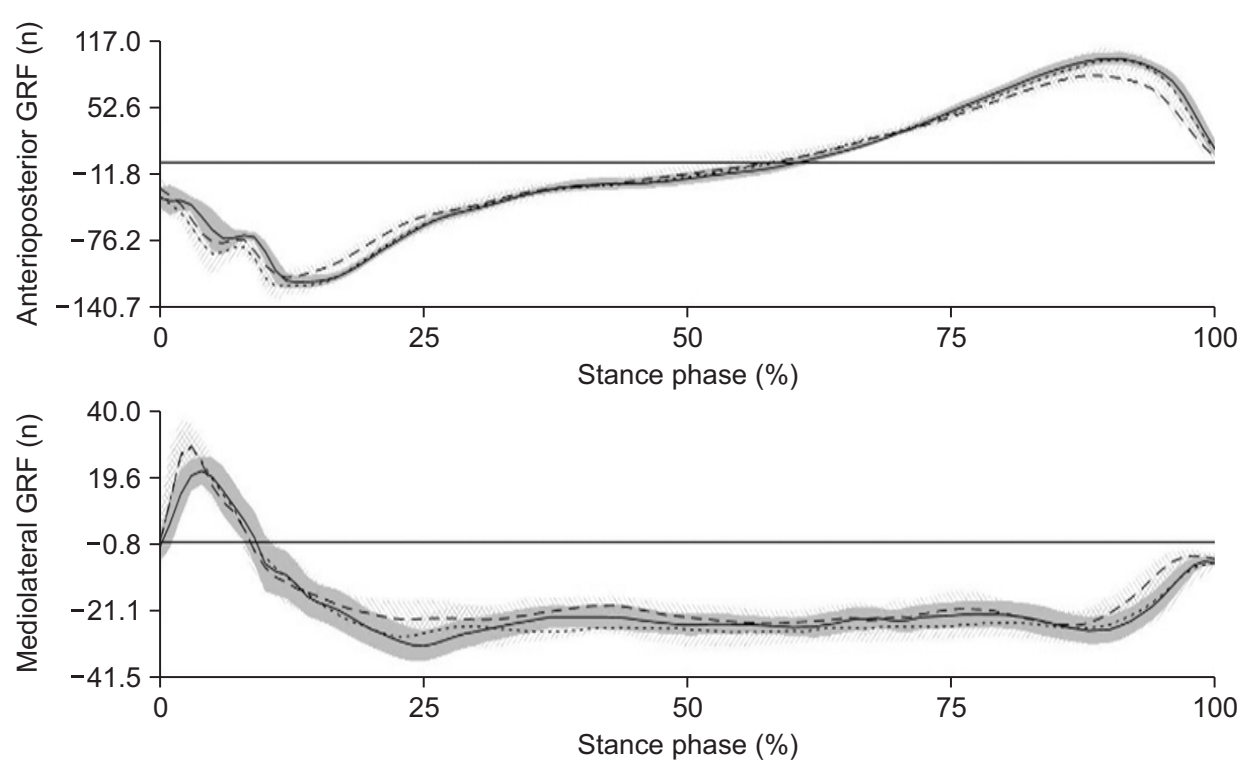

Figure 4. Intra-individual 3D variability of the GRFs during the stance phase. Barefoot GRF (solid line), soft-type orthosis GRF (dashed line), and hard-type orthosis GRF (dotted line) from 24 repetitive trials. The means (lines) and standard deviation (bands) are shown. GRF, ground reaction force. 
was confirmed, the Bonferroni post hoc test was used to verify the pairwise comparison. The GRF values developed bilaterally during each stance phase point (initial and late anteroposterior maximal force, initial and late mediolateral maximal force, first peak force, midstance minimal force, and second peak force) were used for the comparison of each orthosis condition. All analyses were conducted using SPSS version 25.0 (IBM Corp., Armonk, NY, USA). Differences were considered significant at the $\alpha=0.05$ level.

\section{RESULTS}

The mean walking speed in the BF, STO, and HTO conditions of all participants was $1.401 \pm 0.122 \mathrm{~m} / \mathrm{s}, 1.406 \pm 0.131 \mathrm{~m} / \mathrm{s}$, and $1.405 \pm 0.127 \mathrm{~m} / \mathrm{s}$, respectively, and there was no significant difference in walking speed according to the orthosis conditions $(p>0.05)$. There were significant differences in the GRFs between the orthosis conditions in various stance phases (Table 1). The late anteroposterior maximal force during loading (75\%-100\% of the stance phase) and the first vertical peak force of the GRF showed significant differences according to the orthosis conditions $(\mathrm{p}<0.05)$ (Table 1$)$. However, there

Table 1. Repeated-measures ANOVA comparing 3D plane ground reaction force according to each level ( $N=26)$

\begin{tabular}{clcc}
\hline Variables $(\mathrm{N} / \mathrm{kg})$ & \multicolumn{1}{c}{ Level } & $\mathrm{F}$ & $\mathrm{p}$-value \\
\hline IAPMF & Orthosis conditions & 2.517 & 0.094 \\
& Foot sides & 0.521 & 0.467 \\
LAPMF & Interaction effects & 0.524 & 0.459 \\
& Orthosis conditions & 7.120 & $0.012^{*}$ \\
& Foot sides & 0.960 & 0.342 \\
IMLMF & Interaction effects & 1.512 & 0.232 \\
& Orthosis conditions & 0.257 & 0.760 \\
& Foot sides & 1.630 & 0.211 \\
LMLMF & Interaction effects & 2.550 & 0.097 \\
& Orthosis conditions & 0.198 & 0.791 \\
& Foot sides & 1.144 & 0.288 \\
1st VPF & Interaction effects & 0.527 & 0.443 \\
& Orthosis conditions & 3.812 & $0.040 *$ \\
& Foot sides & 0.961 & 0.340 \\
MMF & Interaction effects & 1.524 & 0.224 \\
& Orthosis conditions & 1.942 & 0.152 \\
& Foot sides & 0.897 & 0.363 \\
2nd VPF & Interaction effects & 1.444 & 0.255 \\
& Orthosis conditions & 1.155 & 0.266 \\
& Foot sides & 2.633 & 0.088 \\
& Interaction effects & 2.520 & 0.094 \\
\hline
\end{tabular}

IAPMF, initial anteroposterior maximal force; LAPMF, late anteroposterior maximal force; IMLMF, initial mediolateral maximal force; LMLMF, late mediolateral maximal force; VPF, vertical peak force; MMF, midstance minimal force. ${ }^{*} p<0.05$. were no significant differences in any GRF variables between the foot sides $(\mathrm{p}>0.05)$ (Table 1). There were also no interactive effects between the orthosis conditions and the foot sides in any of the GRF values ( $p>0.05)$ (Table 1).

The specific mean and standard deviation values of each stance phase of the GRF according to the orthosis conditions and foot sides are shown in Table 2. The late anteroposterior maximal force of the GRF showed that the hard-type orthosis condition significantly increased the GRF for the left foot side $(p=0.0008)$ and the right foot side $(p=0.013)$ (Table 2). In addition, the first vertical peak force of the GRF showed that the hard-type orthosis condition significantly increased the GFR when comparing the two orthosis conditions for both foot sides $(\mathrm{p}<0.05)$ (Table 2). However, there were no significant differences in the other GRF variables between the orthosis conditions ( $\mathrm{p}>0.05)$ (Table 2).

\section{DISCUSSION}

This study verified the changes in the various GRF values according to three different foot orthosis conditions using 3D motion analysis in individuals with HVD. The results showed that the HTO condition significantly increased the GRF late anteroposterior maximal force (75\%-100\% stance phase) compared to the STO condition. The first vertical peak force during loading (around the 25\% phase) in the HTO condition was increased significantly compared to the other orthosis conditions. Patients with HVD showed the most noticeable changes in ankle range of motion during walking compared to normal subjects [15]. The repulsive force applied to the feet and toes from the ground during walking is known as a very important variable when evaluating the function of the foot and ankle joints in gait patterns and daily activities such as balance $[16,17]$. The results of this study showed greater GRF changes in the late anteroposterior maximal force and the first peak force in both foot sides when wearing the HTO. Although no previous studies compared these results directly, a previous study evaluated the effect of HVD on gait patterns and foot plantar pressure distribution compared to those of healthy feet using a treadmill installed with an electric pressure sensor mat [18]. There was a significantly higher plantar pressure in the fourth and fifth toes and the second and third metatarsal heads than in the healthy control feet [18]. The previous study results suggested that the application of the HTO in this study elicited 
Table 2. Repeated-measures ANOVA comparing each variable of ground reaction force under the orthosis conditions and foot sides in the stance phase during walking $(\mathrm{N}=26)$

\begin{tabular}{|c|c|c|c|c|c|c|}
\hline Variables (N/kg) & Sides & Barefoot & Soft type orthosis & Hard type orthosis & $\mathrm{F}$ & $\mathrm{p}$-value \\
\hline \multirow[t]{2}{*}{ IAPMF } & Left & $1.83 \pm 0.31$ & $1.87 \pm 0.43$ & $1.95 \pm 0.37$ & 2.693 & 0.090 \\
\hline & Right & $1.79 \pm 0.31$ & $1.84 \pm 0.22$ & $1.90 \pm 0.31$ & 2.468 & 0.093 \\
\hline \multirow[t]{2}{*}{ LAPMF } & Left & $2.12 \pm 0.23$ & $1.96 \pm 0.33$ & $2.17 \pm 0.32$ & 9.452 & $0.000 *$ \\
\hline & Right & $2.14 \pm 0.28$ & $2.05 \pm 0.28$ & $2.19 \pm 0.28$ & 4.787 & $0.013^{*}$ \\
\hline \multirow[t]{2}{*}{ IMLMF } & Left & $0.64 \pm 0.15$ & $0.67 \pm 0.13$ & $0.65 \pm 0.12$ & 0.378 & 0.607 \\
\hline & Right & $0.69 \pm 0.38$ & $0.66 \pm 0.47$ & $0.66 \pm 0.37$ & 0.135 & 0.724 \\
\hline \multirow[t]{2}{*}{ LMLMF } & Left & $0.54 \pm 0.17$ & $0.57 \pm 0.14$ & $0.56 \pm 0.14$ & 0.064 & 0.819 \\
\hline & Right & $0.52 \pm 0.40$ & $0.50 \pm 0.55$ & $0.54 \pm 0.40$ & 0.331 & 0.653 \\
\hline \multirow[t]{2}{*}{ 1st VPF } & Left & $10.50 \pm 0.90$ & $10.96 \pm 1.39$ & $11.05 \pm 1.38$ & 3.481 & $0.038^{*}$ \\
\hline & Right & $10.62 \pm 1.28$ & $10.82 \pm 1.21$ & $11.90 \pm 1.21$ & 3.343 & $0.041 *$ \\
\hline \multirow[t]{2}{*}{ MMF } & Left & $6.68 \pm 1.01$ & $6.93 \pm 1.24$ & $6.76 \pm 1.08$ & 1.402 & 0.256 \\
\hline & Right & $6.87 \pm 0.85$ & $6.94 \pm 0.89$ & $7.10 \pm 0.82$ & 2.483 & 0.094 \\
\hline \multirow[t]{2}{*}{ 2nd VPF } & Left & $11.07 \pm 0.89$ & $11.05 \pm 1.52$ & $11.19 \pm 1.30$ & 0.251 & 0.779 \\
\hline & Right & $11.12 \pm 1.40$ & $11.03 \pm 1.54$ & $11.45 \pm 1.56$ & 2.017 & 0.144 \\
\hline
\end{tabular}

Values are presented as mean \pm standard deviation. IAPMF, initial anteroposterior maximal force; LAPMF, late anteroposterior maximal force; IMLMF, initial mediolateral maximal force; LMLMF, late mediolateral maximal force; VPF, vertical peak force; MMF, midstance minimal force. ${ }^{*} \mathrm{p}<0.05$.

more medial transfer of foot plantar pressure, which resulted in more effective forward propulsion in the terminal stance phase during walking. The results of this study showed no significant change in any GRF variables between the BF and STO conditions. This may be because the STO was not able to push the first metatarsophalangeal joint inward enough to change the hallux valgus angle due to the characteristics of the soft material of the STO compared to the rigid material of the HTO.

In this study, most of the GRF variables were not affected when applying the foot orthoses except for the late anteroposterior maximal force and the first vertical peak force. Many previous studies reported that there were no significant differences in the spatiotemporal gait parameters between HVD and healthy feet [11,19-21] and a previous study reported a difference in GRF between HVD and healthy feet. Therefore, HVD seems not to affect the overall walking pattern [18]. Although in a forefoot deformity such as HVD, compensation occurs in the foot or ankle joint, the overall gait parameters such as step width, step length, and single-limb support are not affected.

Most of the previous studies verifying the changes in GRF following HVD interventions were performed by surgical treatments of the foot or ankle such as scarf osteotomy and first metatarsophalangeal arthrodesis [22,23]. However, this study applied conservative intervention, not a surgical treatment for HVD, to verify the effect on the GRF through more objective and quantitative 3D motion analysis and a force platform. Therefore, in this study, significant changes in the GRF could be verified through various types of foot orthoses. However, this study had some limitations. Although the participants had
HVD, this study was executed on healthy individuals who had no pain or discomfort in their feet and toes. Therefore, it is difficult to generalize these results to those with painful HVD. This study was a cross-sectional study design and did not verify the long-term effects of foot orthoses for HVD. Considering the difficulty in the analysis, the verification of kinetic variables other than the GRF occurring in various lower limb joints such as the hip, knee, and ankle was not conducted. Further studies are needed to verify the effects of the various interventions on the kinetics and kinematic changes over time in individuals with HVD.

\section{CONCLUSIONS}

This study examined the effects of 3D dynamic GRF by applying two different foot orthoses and barefoot conditions when walking in 26 individuals with HVD. The results showed significant effects in the late anteroposterior maximal force and the first vertical peak force of the GRF when wearing the HTO during walking. However, the HTO had the disadvantages of being uncomfortable when wearing and made it difficult to wear shoes due to the characteristics of the hard material. Therefore, it is necessary to develop a new type of foot-toe orthosis for HVD that addresses the disadvantages of HTOs and STOs.

\section{ACKNOWLEDGEMENTS}

This research was supported by the National Research Foun- 
dation of Korea (NRF) grant funded by the Ministry of Science and ICT (No. 2018R1C1B5042645).

\section{CONFLICTS OF INTEREST}

No potential conflict of interest relevant to this article was reported.

\section{REFERENCES}

1. Vanore JV, Christensen JC, Kravitz SR, Schuberth JM, Thomas JL, Weil LS, et al.; Clinical Practice Guideline First Metatarsophalangeal Joint Disorders Panel of the American College of Foot and Ankle Surgeons. Diagnosis and treatment of first metatarsophalangeal joint disorders. Section 1: hallux valgus. J Foot Ankle Surg 2003;42(3):112-23.

2. Doty JF, Harris WT. Hallux valgus deformity and treatment: a three-dimensional approach. Foot Ankle Clin 2018;23(2):27180

3. Ferrari J. Hallux valgus (bunions). Clin Evid 2002;(7):983-91.

4. Baravarian B, Ben-Ad R. Revision hallux valgus: causes and correction options. Clin Podiatr Med Surg 2014;31(2):291-8.

5. Perera AM, Mason L, Stephens MM. The pathogenesis of hallux valgus. J Bone Joint Surg Am 2011;93(17):1650-61.

6. Coughlin MJ, Jones CP. Hallux valgus: demographics, etiology, and radiographic assessment. Foot Ankle Int 2007;28(7):75977.

7. Zirngibl B, Grifka J, Baier C, Götz J. [Hallux valgus: etiology, diagnosis, and therapeutic principles]. Orthopade 2017;46(3):283-96. German

8. Kim MH, Yi CH, Weon JH, Cynn HS, Jung DY, Kwon OY. Effect of toe-spread-out exercise on hallux valgus angle and crosssectional area of abductor hallucis muscle in subjects with hallux valgus. J Phys Ther Sci 2015;27(4):1019-22.

9. Shih KS, Chien HL, Lu TW, Chang CF, Kuo CC. Gait changes in individuals with bilateral hallux valgus reduce first metatarsophalangeal loading but increase knee abductor moments. Gait Posture 2014;40(1):38-42.

10. Menz HB, Lord SR. The contribution of foot problems to mobility impairment and falls in community-dwelling older people. J Am Geriatr Soc 2001;49(12):1651-6.

11. Deschamps K, Birch I, Desloovere K, Matricali GA. The impact of hallux valgus on foot kinematics: a cross-sectional, com- parative study. Gait Posture 2010;32(1):102-6.

12. Tang SF, Chen CP, Pan JL, Chen JL, Leong CP, Chu NK. The effects of a new foot-toe orthosis in treating painful hallux valgus. Arch Phys Med Rehabil 2002;83(12):1792-5.

13. Chadchavalpanichaya N, Prakotmongkol V, Polhan N, Rayothee P, Seng-Iad S. Effectiveness of the custom-mold room temperature vulcanizing silicone toe separator on hallux valgus: a prospective, randomized single-blinded controlled trial. Prosthet Orthot Int 2018;42(2):163-70.

14. Collins TD, Ghoussayni SN, Ewins DJ, Kent JA. A six degreesof-freedom marker set for gait analysis: repeatability and comparison with a modified Helen Hayes set. Gait Posture 2009;30(2):173-80.

15. Klugarova J, Janura M, Svoboda Z, Sos Z, Stergiou N, Klugar M. Hallux valgus surgery affects kinematic parameters during gait. Clin Biomech (Bristol, Avon) 2016;40:20-6.

16. Ballas R, Edouard P, Philippot R, Farizon F, Delangle F, Peyrot N. Ground-reactive forces after hallux valgus surgery: comparison of Scarf osteotomy and arthrodesis of the first metatarsophalangeal joint. Bone Joint J 2016;98-B(5):641-6.

17. Barbee CE, Buddhadev HH, Chalmers GR, Suprak DN. The effects of hallux valgus and walking speed on dynamic balance in older adults. Gait Posture 2020;80:137-42.

18. Hofmann UK, Götze M, Wiesenreiter K, Müller O, Wünschel M, Mittag F. Transfer of plantar pressure from the medial to the central forefoot in patients with hallux valgus. BMC Musculoskelet Disord 2019;20(1):149.

19. Nix SE, Vicenzino BT, Collins NJ, Smith MD. Characteristics of foot structure and footwear associated with hallux valgus: a systematic review. Osteoarthritis Cartilage 2012;20(10):1059-74.

20. Menz HB, Lord SR. Gait instability in older people with hallux valgus. Foot Ankle Int 2005;26(6):483-9.

21. Mickle KJ, Munro BJ, Lord SR, Menz HB, Steele JR. Gait, balance and plantar pressures in older people with toe deformities. Gait Posture 2011;34(3):347-51.

22. Kia C, Yoshida R, Cote M, DiVenere J, Geaney LE. First metatarsophalangeal contact properties following proximal opening wedge and scarf osteotomies for hallux valgus correction: a biomechanical study. Foot Ankle Int 2017;38(4):430-5.

23. Barouk LS. Scarf osteotomy for hallux valgus correction. Local anatomy, surgical technique, and combination with other forefoot procedures. Foot Ankle Clin 2000;5(3):525-58. 